\title{
Students List FRs Chronologically and DPs Spatially, and Need to Integrate FRs Functionally and DPs Physically
}

\author{
Masayuki $\mathrm{Nakao}^{1, *}$, and Kenji Iino ${ }^{1}$ \\ ${ }^{1}$ School of Engineering, The University of Tokyo, Japan
}

\begin{abstract}
The authors have students draw Functional Requirement (FR) - Design Parameter (DP) charts for their free subject design assignments before teaching them Axiomatic Design (AD) in detail. The students cannot list all important FRs and DPs even with Zigzag thinking between FR and DP from higher level to lower; then, they tend to list FRs chronologically like flowcharts and DPs spatially like bill of material. The strategy, however, often results in different counts of FRs and DPs and a number of interferences among them. When this happens, we lead the students to carry out the thinking processes of functional integration of FRs and physical integration of DPs so they can reach a regular matrix for the design equation. Once regularization is reached, the students can apply independence axiom to improve their designs.
\end{abstract}

\section{Introduction: Problems in defining FRs for design projects}

Engineers start design thinking about some new solutions to meet social needs (design for creation) or, upon recognizing problems in conventional physical products or organizational systems, to improve them (design for improvement). In either case, taking on the design in a rush by gathering what is available on hand to put together a quick and imperfect solution is not recommended. The key to success is to put down the prototyping tools, organize the variety of concepts that construct the design, and define the design that is about to be built.

In our department, we assign design projects to undergraduate and graduate students for which they are free to select their own project goals. [1] The instructors hope that the students will come up with designs for creation that can lead to patents, however, their deep search into the Internet often leads to imitate something good and carry out design for improvements at the end. Our instructions have the students start on their designs by first defining their functional goals before they start physical prototyping. We, however, keep the definitions at the minimum, so the students do not lose the desire that they want to start prototyping immediately. The minimum definition is a set of functional requirement (FR) without planning on any design parameter (DP), that is, "FR first." In fact, FR is the very indicator that evaluates the new DP constructed by the designer, and at the same time it is the index for the customer to evaluate the design itself.
In defining a design, we must first extract and select phrases that express elements literately to use in the conceptual design definition. We then analyse those concepts as they are, organize them and redefine them. In this difficult analysis phase, the instructors need to help the students in defining these phrases and concepts; thus, all that are left for the students to work on are only to realize the concepts. At these tasks, Japanese engineering students can notice that they are not good at handling such abstract concepts. They are particularly weak in dealing with FRs without physical substance.

Fig. 1 shows the phased methodology of defining a design we teach. The authors translated the Suh's textbook on Axiomatic Design (AD) [2] into Japanese in 2004; we started to teach the undergraduate students to define their designs as the textbook instructed. The class, however, didn't work well because our Japanese design curriculum imported from England in 1888 was too classic, and the students had firstly learned artifacts; for example, screws, gears, engines, wings and so on, namely, "DP first." The students were already imprinted that design thinking was only a physical implementation of DPs. So we added some pre-processes like mind mapping or work breakdown structuring to think about customer need $(\mathrm{CN})$ and FR before teaching $\mathrm{AD}$. The students have no experience to use words, not $\mathrm{CAD} / \mathrm{CAM} / \mathrm{CAE}$, in a design class. So we let the student take on a project only with words. They want to define the design literally, but don't have enough volume of words. They are like a chef who wants to cook the dinner, but doesn't have enough materials such as meats, fishes, salt, etc.

\footnotetext{
* Corresponding author: nakao@hnl.t.u-tokyo.ac.jp
} 


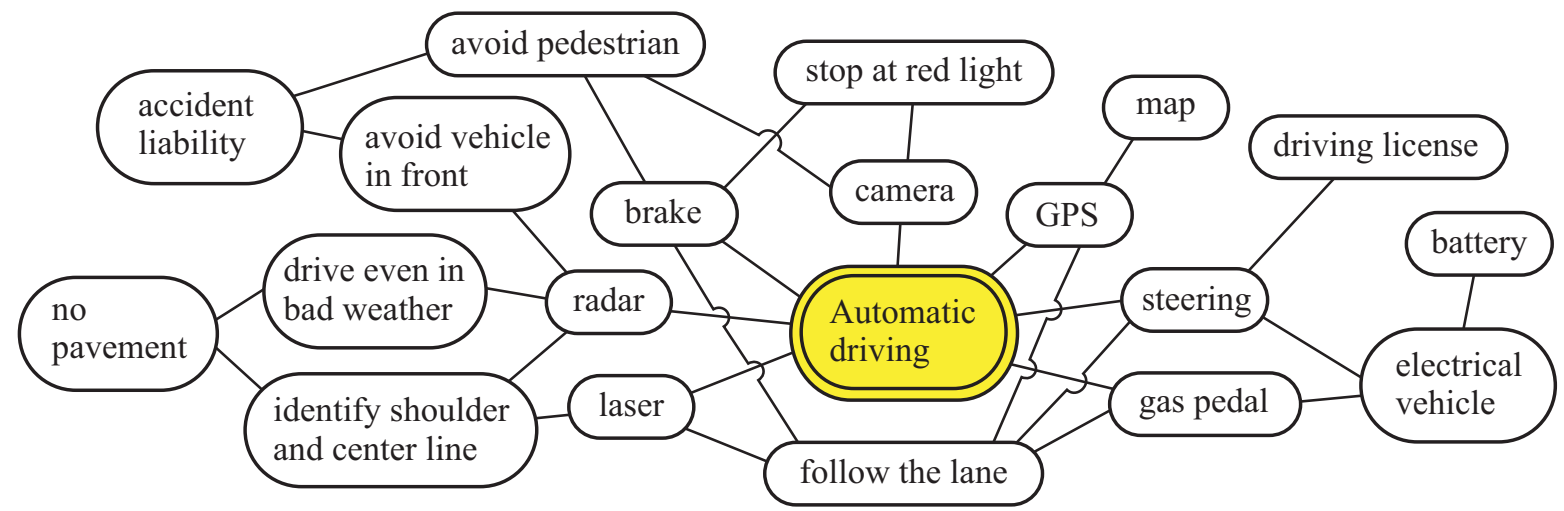

(a) Mind map: List out elements of design concepts as they come to mind

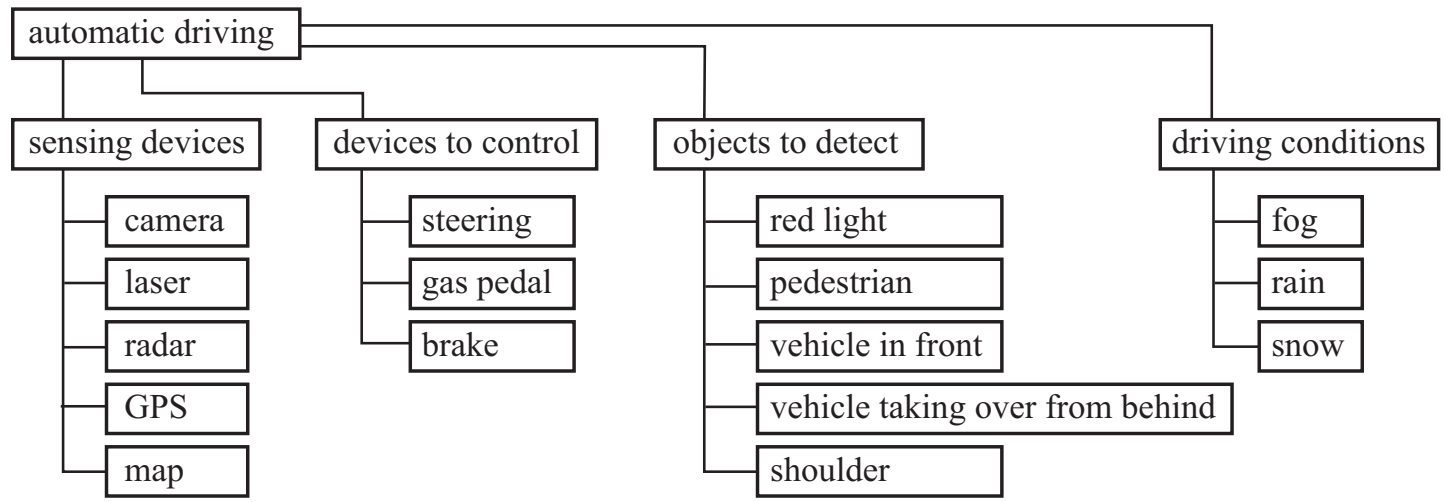

(b) Work breakdown structure: Collect similar concepts and bring them all out

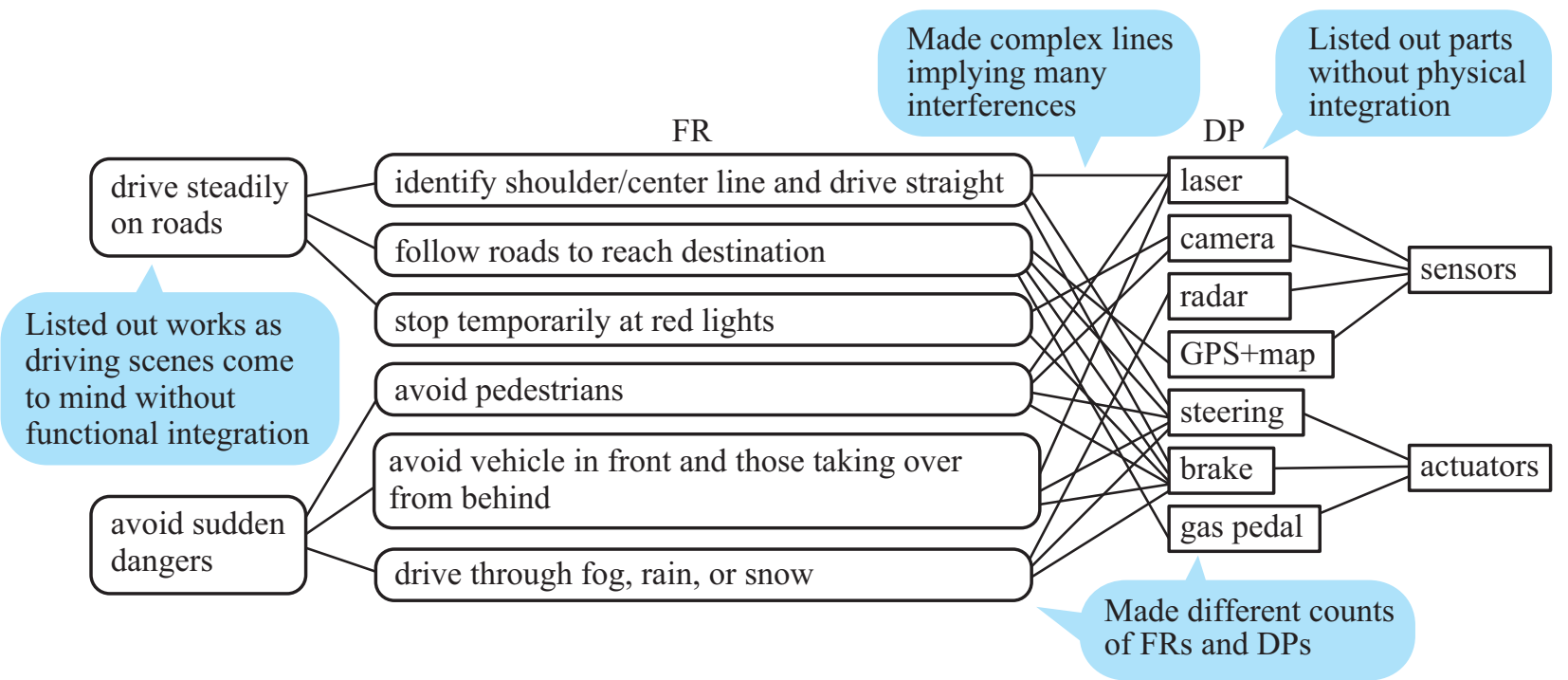

(c) FR-DP chart: Group FRs and DPs and make connections for relations

$$
\begin{aligned}
&\left\{\begin{array}{l}
\text { follow roads to reach destination } \\
\text { avoid shoulder, center line, vehicle in front and pedestrians } \\
\text { stop at red lights and stop signs } \\
\text { drive through fog, rain, or snow }
\end{array}\right\}=\left[\begin{array}{llll}
\mathrm{X} & 0 & 0 & 0 \\
\mathrm{X} & \mathrm{X} & 0 & 0 \\
\mathrm{X} & 0 & \mathrm{X} & 0 \\
\mathrm{X} & 0 & 0 & \mathrm{X}
\end{array}\right]\left\{\begin{array}{l}
\text { GPS }+ \text { map }+ \text { actuators } \\
\text { laser (time of flight) } \\
\text { camera (image processing) } \\
\text { radar (doppler) }
\end{array}\right\} \\
& \text { Made design matrix regular }
\end{aligned}
$$

(d) Design equation: Integrate FRs functionally and DPs physically

Fig. 1. Methods for manipulating design concepts. 
For example, the project is to design automate driving of a car as shown in Fig. 1(a). When told to list elements for conceptual design, they would start thinking about mechanisms (DPs) for automatic control like "camera," "laser," "radar," "GPS," "steering wheel," and "brake." Students today rely on their searches on the Internet and the searched information easily their work.

Following the next instruction to identify functions (FRs) related with each DP, they list, for example, "follow roads," "stop at red lights," "avoid vehicle in front," "avoid pedestrians," and "drive through poor weather." Listing out all these elements, however, is difficult. Nobody knows if these are all or not. Students always miss some intrinsic FRs.

Fig. 1(a) shows a mind map, often used for hitting all the needed elements. The method pulls out all the elements instinctively in a manner like pulling objects attached to a string one after another, and sets the concepts in nodes and draws lines for relations among them. At least, the number of nodes should be 20 or more.

The next step is to group the concepts and to avoid anything from slipping the mind. The systematic approach of work breakdown structure is effective (Fig. 1(b)). The elements here are still not categorized into design concepts; namely, FRs, DPs, constraints, effects, risks and so on are mixed together. Thompson introduced kinds of functional information [3] and procedural errors in the definition of FR [4]. According to her classification, the authors could often find the errors of "mixing FRs with other types of requirement information" in the design class as mentioned at 3.4 later; FRs were mixed, for example, with non-functional requirements (durable, easy to use, etc.), or with input constraints (cost, weight, operating temperature range, etc.). Thompson pointed out that even Suh [2] or Brown [5] had made the errors of mixing FRs with input constraints.

Our design education has emphasis on recognizing FRs and we have the students pull out verbs for FRs and nouns for DPs and have them draw FR-DP charts like Fig. 1(c) shows. The instructors tell the students to list out all the FRs and DPs, and the students tend to list FRs chronologically like flowcharts and DPs spatially like bills of material. Consequently, each FR has some DPs which correspond or relate to the FR, but not the only one DP which satisfies the FR.

Of course, the students should draw the FR-DP chart in a manner following Suh's Zigzag thinking [2] by traversing the chart. At first, they should set a higher FR, then set the higher corresponding DP, then go down a lower FR, and set the lower DP. That is to alternate between FR and DP in setting the concepts in the order of abstraction, then both sides will have the same number of FRs and DPs with a symmetric tree structure, and the design matrix will be regular (regular: square with an inverse matrix; determinant is non-zero). We teach the students this Zigzag methods; the students, however, still miss some important FRs, or want to recover the shortage of FRs from the DPs reversely. In results, Fig. 1(c) shows complex lines between FRs and DPs. The lines implied many interferences of FRs.
The last phase is to derive design equation shown in Fig. 1(d) for AD. In the previous phase, the students draw the complex lines in Fig 1(c). As the numbers of FRs and DPs do not match, the design matrix should not be regular. As lines that connect FRs with DPs make a number of intersections, the design should not be uncoupled. We need an exact one-to-one relation between FRs and DPs.

Generally, the students make their efforts in listing up all the design elements, and identify FRs chronologically and DPs spatially. The trend is not special to students who are novices in design, but even experienced engineers at corporations, without any experience of defining the products, have the same tendency. Their recoverable method, nevertheless, meets their purposes of identifying all the FRs and DPs. Then, they start to try the Zigzag method with the collected FRs and DPs, and make a regular and uncoupled design matrix. Fig. 1(d) shows the regular and lower triangular design matrix. All four FRs were the higher FRs; this design didn't need the Zigzag method to find lower FRs. To make the square matrix, the instructors tell the students to integrate FRs functionally and DPs physically to condense into enough small numbers of FRs and DPs.

In applying $\mathrm{AD}$, it is ideal to match the counts of FRs and DPs and arrange them in the same tree structure with the Zigzag method. To cope with the situation, the thinking processes of functional integration for FRs and physical integration for DPs is also effective. This paper introduces this functional integration and physical integration with actual design cases.

\section{Actual cases of design definition}

Defining the FR is clearly more difficult with "design for creations" compared to "design for improvement." The former does not yet realize the new DPs, in the conceptional design process. Thus, FRs cannot be inferred from DPs and the students have to express invisible FRs with words alone.

We give the undergraduate students free subject design assignments for Stirling engines or Internet of Things (IoT), we hope to see "designs for creations" that are worth patenting, but we know that the results may end up in "design for improvement" because about 30 hours of its project term is too short to invent something and define the right set of FRs and DPs.

The problem with these assignments for undergraduate students are the process where they tend to deploy FRs chronologically and DPs spatially. Thus, the design equation for AD turns out non-regular. This is the time when the instructors have to support the students in their analyses to carry out functional integration for FRs and physical integration for DPs. This chapter discusses our work with three actual cases.

Throughout the academic years, we constantly see students' researches for degrees of bachelor, master's, and doctoral. Even such a novel creative academic thesis reveals that most of the work are dependent on past researches, it has only a few DPs that are worth insisting 
priority and creativity when we have the students draw FR-DP charts. In 2018, 42 graduate students (51\%) out of 83 could draw the right FR-DP charts and indicate the prior and creative DPs; 24 students (29\%), however, made the errors of "mixing the FRs of the designer" (the project budget, the project dead line, etc.) which should be constraints according to Thompson [3]. These 51\% students had already published their own academic papers; they could pick up FRs functionally from the purpose of the research in the papers.

\subsection{Case 1: Fan design}

Fan is a simple mechanical design without any control computers, probably ideal to have the students identify FRs. The authors asked junior students in the design class to make an FR-DP chart and a design equation of the fan. Fig. 2(a) shows an example of the FR-DP chart which most of them made. We can see complex lines between FRs and DPs.

This student chronologically extracted the FRs thinking about a customer that just made a purchase. The student first thought about the operations of "connecting to AC power" and "pressing the airflow switch." The student also came up with the operations of "pressing the oscillation switch" and "turning the shutoff timer" when

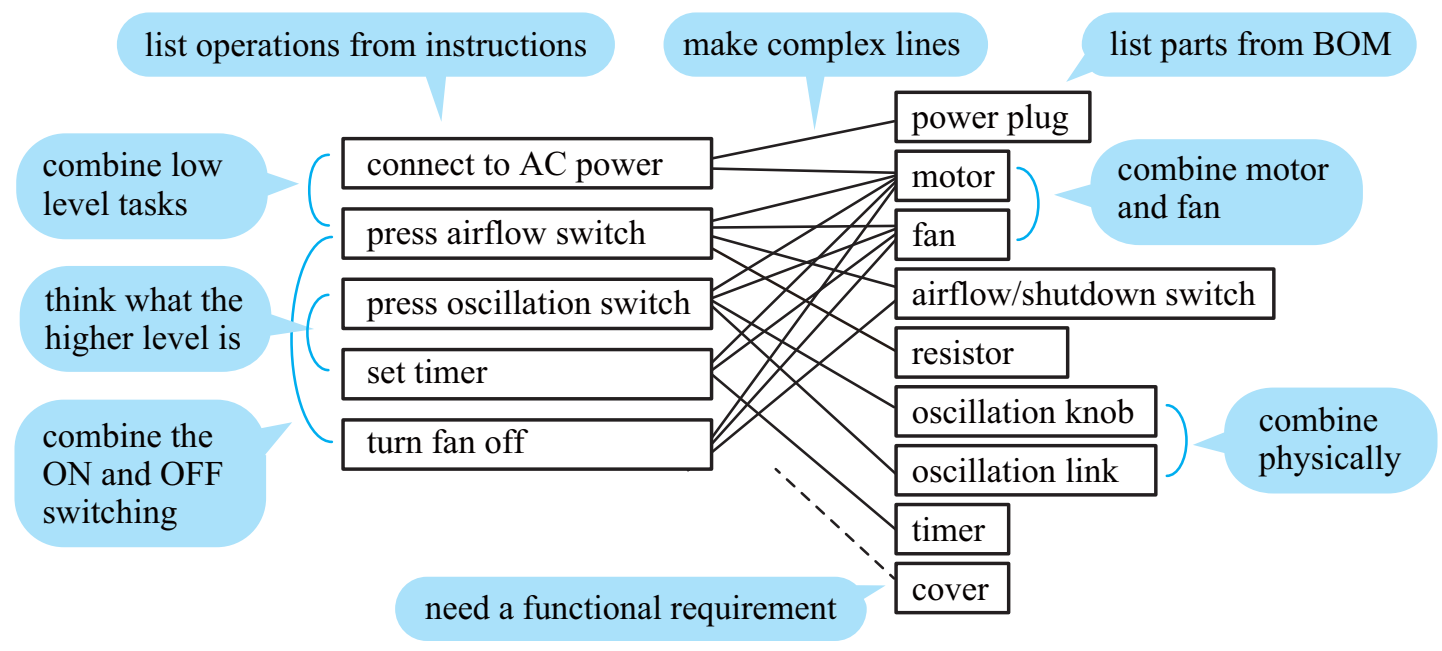

(a) FR-DP chart by chronologically listing FRs and spatially listing DPs

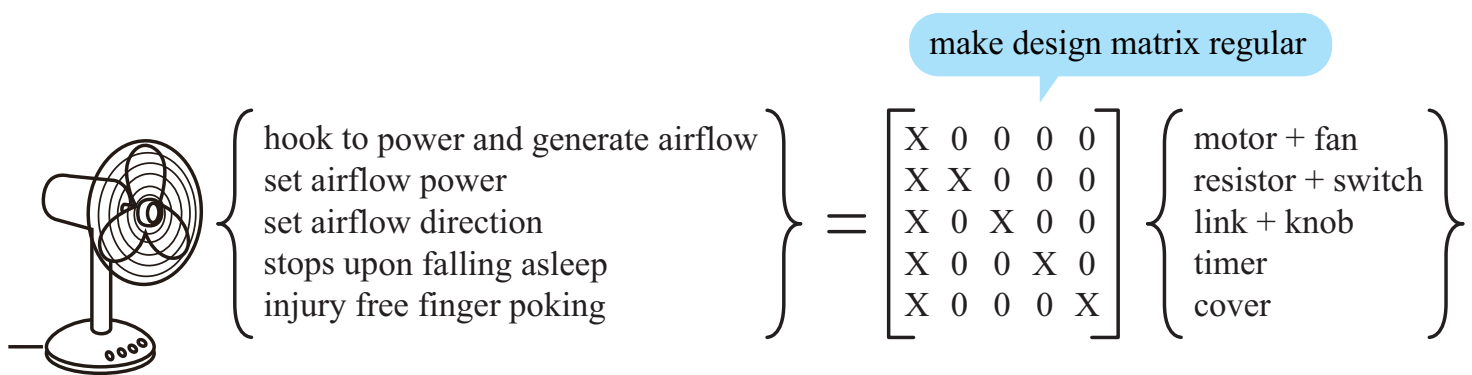

(b) Design equation that integrated FRs functionally and DPs physically

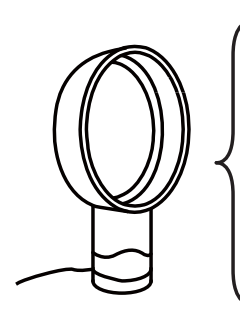

$\left\{\begin{array}{l}\text { hook to power and generate airflow } \\ \text { set airflow power } \\ \text { set airflow direction } \\ \text { stops upon falling asleep } \\ \text { injury free finger poking } \\ \text { arrange blades invisible }\end{array}\right\}=\left[\begin{array}{cccccc}\mathrm{X} & 0 & 0 & 0 & 0 & 0 \\ \mathrm{X} & \mathrm{X} & 0 & 0 & 0 & 0 \\ \mathrm{X} & 0 & \mathrm{X} & 0 & 0 & 0 \\ \mathrm{X} & 0 & 0 & \mathrm{X} & 0 & 0 \\ \mathrm{X} & 0 & 0 & 0 & \mathrm{X} & 0 \\ \mathrm{X} & 0 & 0 & 0 & 0 & \mathrm{X}\end{array}\right]$

(c) Design equation of a bladeless fan

Fig. 2. Fan design. 
going to bed.

On the other hand, the bill of material led to spatial decomposition of "power plug," "motor," "fan," "airflow/shutdown switch," "resistor," "oscillation knob," "oscillation link," "timer," and "cover." They can not represent a DP as something to satisfy an FR.

The student then connected related FRs on the left and DPs on the right with lines. The first FR of "connect to AC power" related to DPs of "power plug" and "motor." The lines had many branches and crossings, however, they were inerasable. On the other hand, the student recognized that the last DP of "cover" did not have a line to an FR and added the FR of "prevent finger poking by children."
Following our instruction, the students then started functional integration of FRs and physical integration of DPs. "Motor" and "fan" connected to the same FR, thus, the student physically integrated them. Similarly, "oscillation knob" and "oscillation link" combined as well.

The FR of "turn fan off" related to DPs of "motor," "fan," and "airflow/shutdown switch" and so did the another FR of "press airflow switch." The student then integrated these two FRs into a single FR of "set airflow power." The FR of "connect to AC power" was a lower FR to the FR of "press airflow switch," thus, the two FRs could integrate to the one FR of "hook to power and generate airflow."

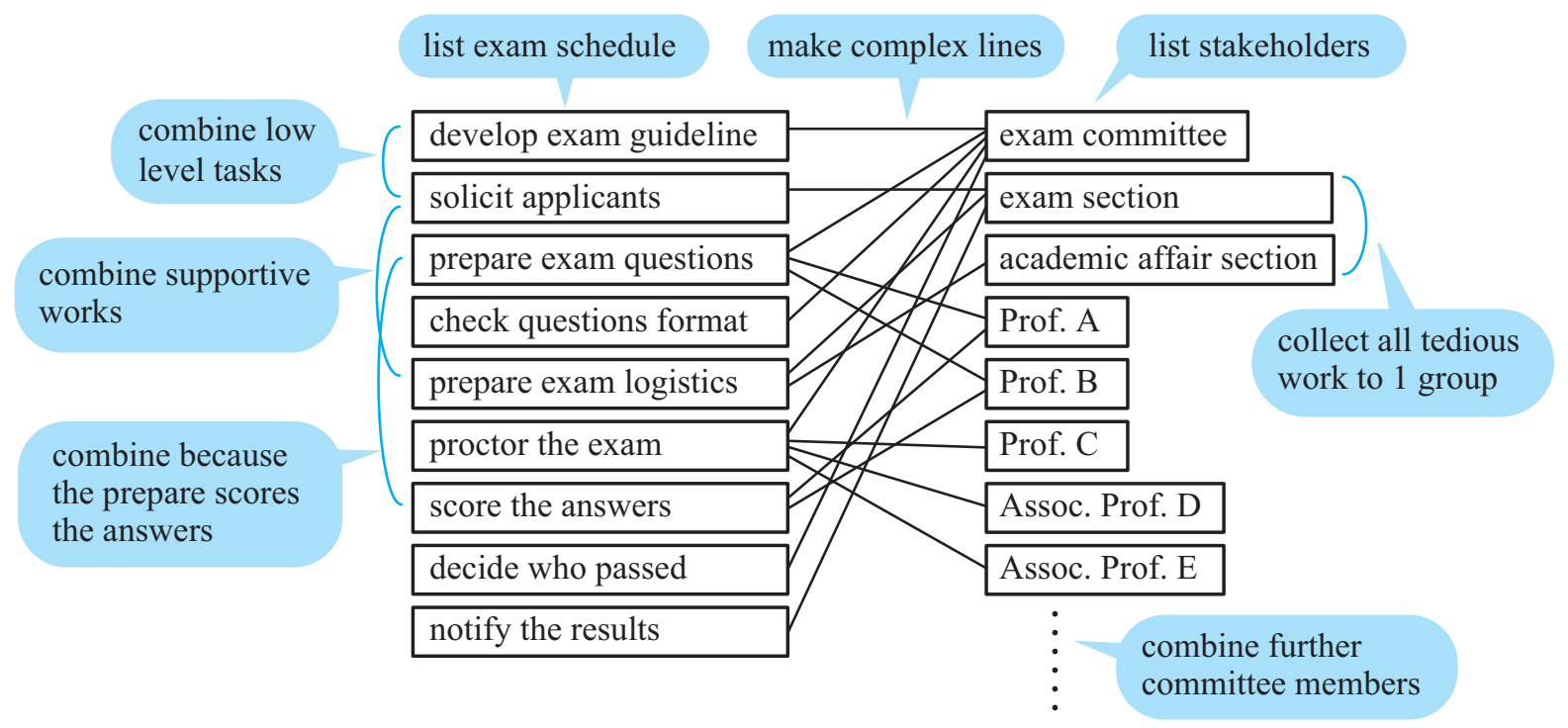

(a) FR-DP chart by chronologically listing FRs and spatially listing DPs

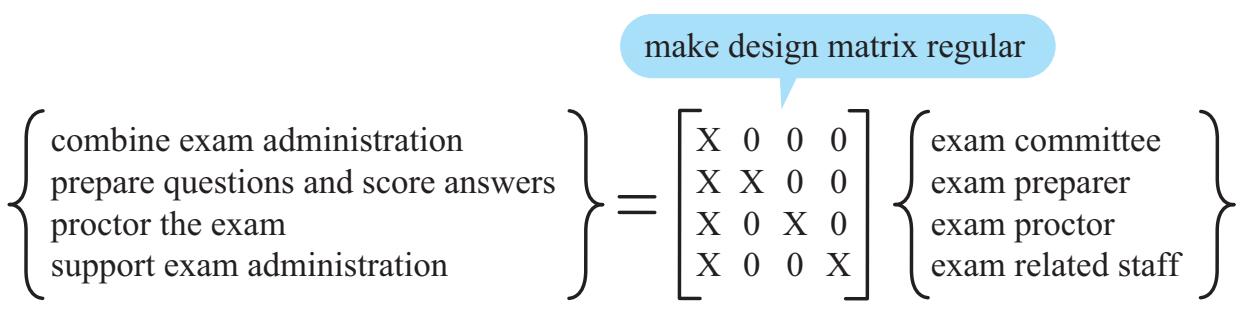

(b) Design equation that combined FRs functionally and DPs physically

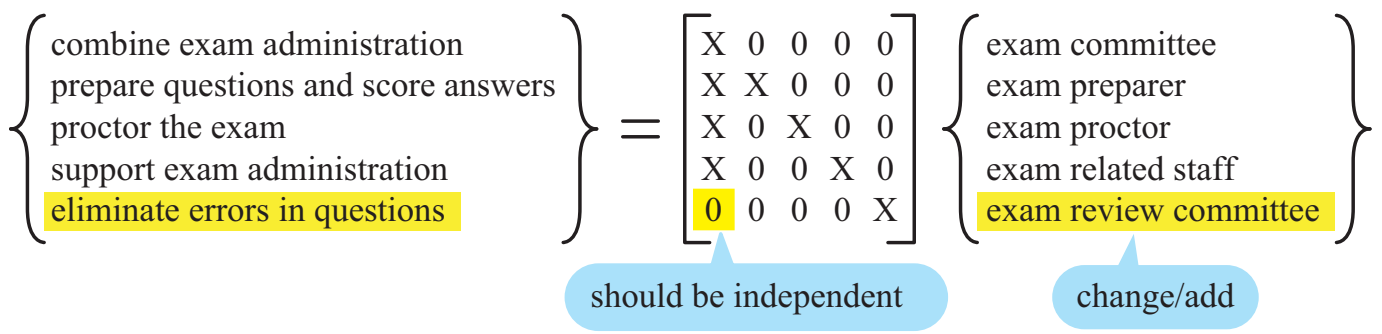

(c) Design equation for eliminating errors in questions

Fig. 3. Planning administration of admission exam. 
Figure 2(b) is the design equation after these changes. It means a decoupled design, and the DP of "motor + fan" needs to be decided at first. FRs were functionally organized and each corresponded to a physically integrated DP. The design matrix shows that all FRs related to the first DP of "motor + fan" with all first column elements being X; "motor + fan" was the key component of the fan.

Once the design equation is polished up, we could see how design for improvements affect the product. For example, the "bladeless fan" in Fig. 2(c) only changed the "cover" to a "ring-shaped blower." This change in how the FR of "prevent finger poking" was realized, however, does not explain the explosive sale by order of magnitude. We can then add the new FR of "arrange blades invisible." We inferred that this FR surprized the consumers and generated the next generation value of a fan.

One of the authors, Nakao has a bladeless fan in his office, however, the shortcoming is that the noise of the airflow is large and annoying. We should set quietness as a constraint in a way similar to cost or durability.

\subsection{Case 2: Entrance exam administration}

Administration for an entrance exam is a good simple task for designing an organization. Figure 3(a) shows an FR-DP chart made by a junior student who wore a professor's hat.

The student listed the FRs following the time sequence of reaching the day of the exam. The FRs were chronologically set to "develop exam guideline," "solicit applicants," "prepare exam question," "check questions format," and so on. DPs, on the other hand, started by listing up the stakeholders spatially. They were "exam committee," "exam section," "academic affair section," "Prof. A," and so on.

Connecting related elements with lines produced a complex chart similar to the previous fan example, thus, the student needed some integration work. The FRs, for example, of "prepare exam questions" and "score the answers" were carried out by the same person, thus, they could be functionally integrated to "prepare questions and score answers." DPs of "exam section" and "academic affair section," together, were in charge of the FR of supportive administration, thus, they could physically integrate to "exam related stuff."

Figure 3(b) shows the design equation after integrating FRs into 4 categories. The four FRs were "combine exam administration," "prepare questions and score answers," "proctor the exam" and "support exam administration." Elements in the first row of the design matrix are all X meaning all FRs were affected by the leader "exam committee."

Once the design equation was organized to this level, the effect of "design for improvement" was foreseeable, like in the case of bladeless fan. For example, a newspaper recently made large noise about mistakes in entrance exam questions and the University of Tokyo set a new FR of "eliminating errors in questions." The School of Engineering assigned a young faculty member with a flexible thinking as "exam review committee" and had this member challenge the questions. Some errors in questions were identified every year before the exam. During this checking, it is important that the exam review committee looks at the questions independently without knowing "who made the questions." If the member starts to worry like "If I find an error in the work by that big professor, that may influence my future," errors in the questions may not surface.

\subsection{Case 3: Umbrella that follows the owner}

Next, we will introduce a case from free subject design assignment. A student group designed an umbrella that followed movement of the owner as the sketch in Fig. 4(a) shows in the next page. They first used a drone but the noise from the four nearby rotors was too big to the ears. They then generated lifting force with a helium balloon to counter the dead weight of the machine. The design also used two small motored propellers powered by small batteries to linearly move the balloon to follow the owner and make yawing motions to the left and right.

Fig. 4(b) shows the FR-DP chart. The students forced a one-to-one correspondence among the FRs and DPs, however, the tree shows the left half FR and right half DP trees had different structures. This mismatch was caused by defining a DP for each part. Fig. 4(c) shows the prototype in motion. The instructors lead the students to integrate FRs and DPs. The number of FRs after the integration might to be 5 or less.

The umbrella followed the owner with a camera finding his head and Raspberry $\mathrm{Pi}$ reasoning the propeller activation. The owner wore a red cowboy hat for better recognition, however if the head moved too far away from the camera, the camera was lost, and the next step under discussion is to use a fisheye lens. Also, the double propeller design caused the balloon to roll and pitch, and the stable floating would last for only a short time. A tail wing is also under evaluation to prevent rolling and pitching.

Fig. 4(d) is the design equation we constructed after the results and discussion. In addition to the FR of "control umbrella to follow owner," we added the FR of "prevent pitching and rolling" and the corresponding DP of "tail wing."

\section{Discussions: Teaching methods to make design equation right for design projects}

The above three case studies clarified the processes of identifying all FRs and DPs and then applying integration to regularize the design matrix. We can collect so many FRs, but for the analysis using AD, the FRs might be condensed into about 5. Whatever the design is, the same process can lead to a good uncoupled or decoupled design matrix, however, this work is still challenging for students. We introduce our teaching methods for design projects. 


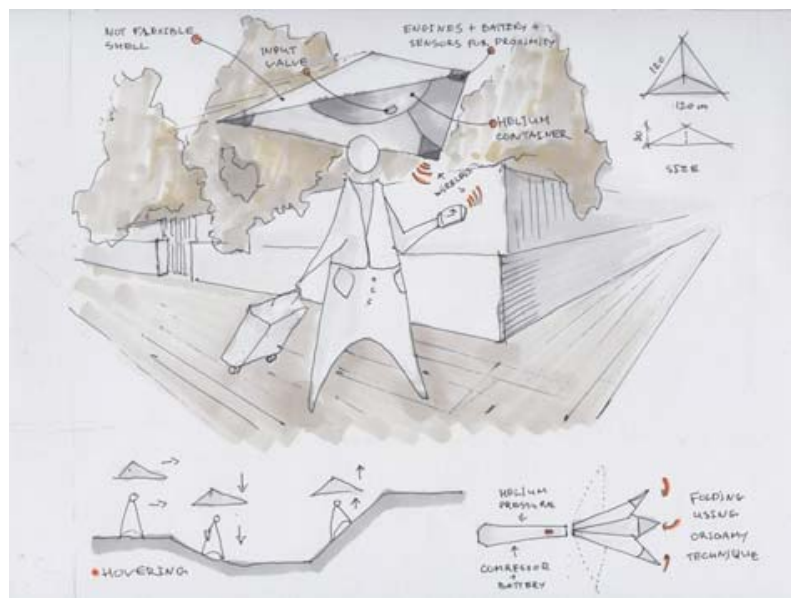

(a) Conceptual sketch of the design

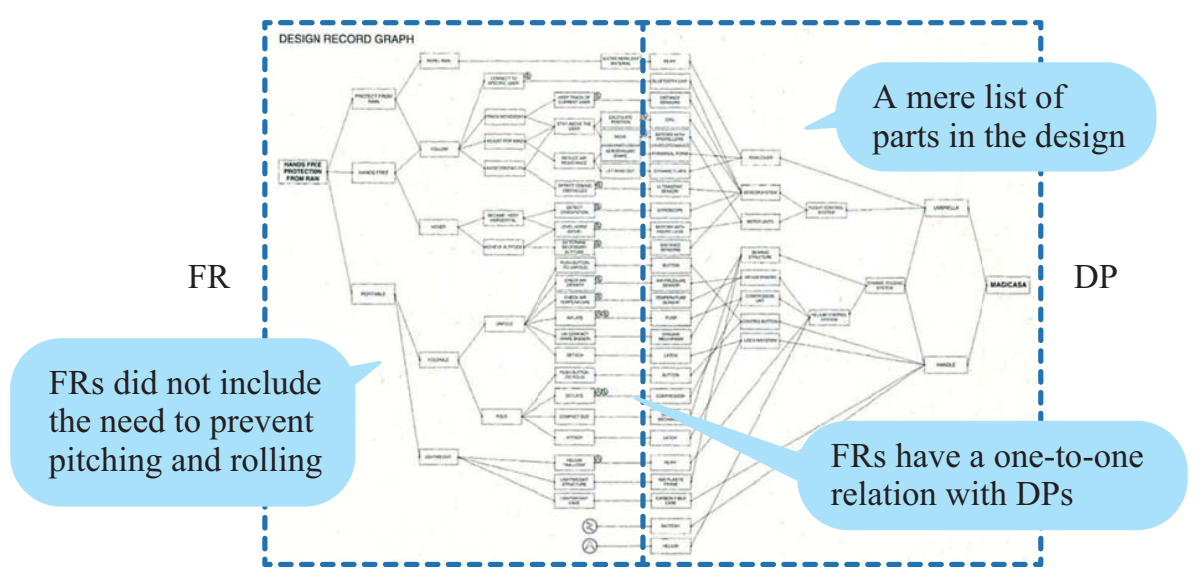

(b) FR-DP chart

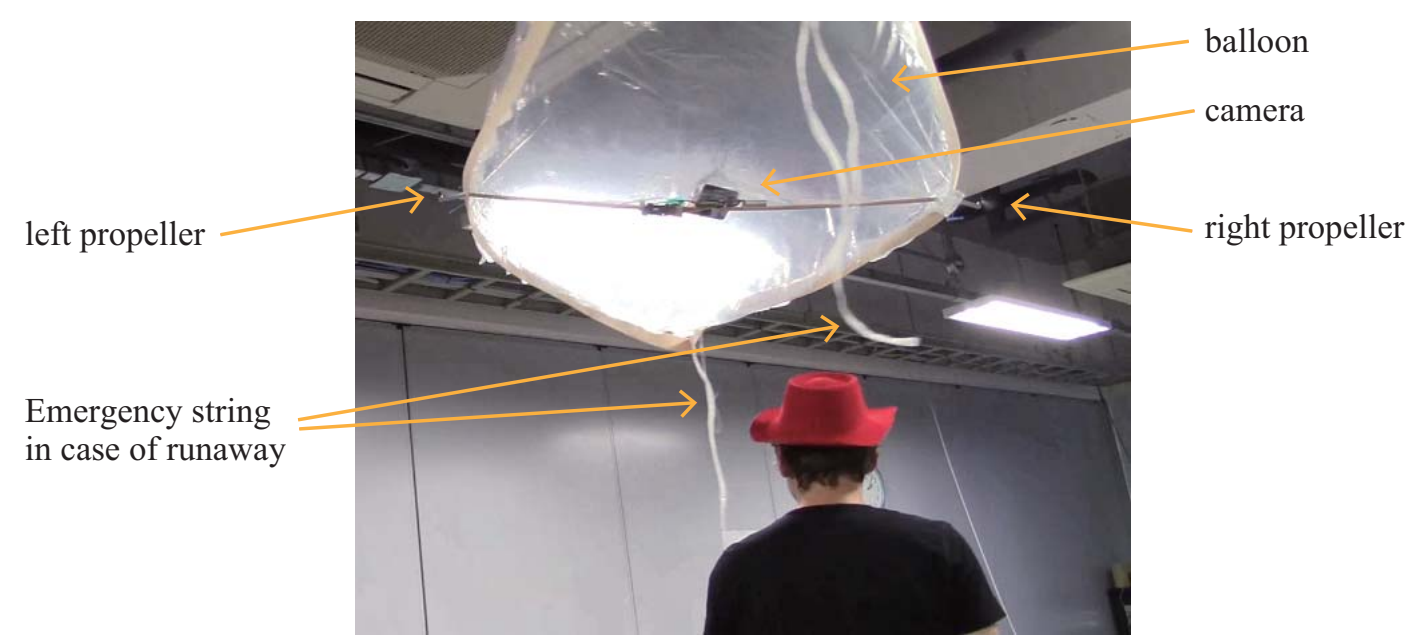

(c) Actual prototype (camera finds the red hat and follows it by controlling the left and right propellers)

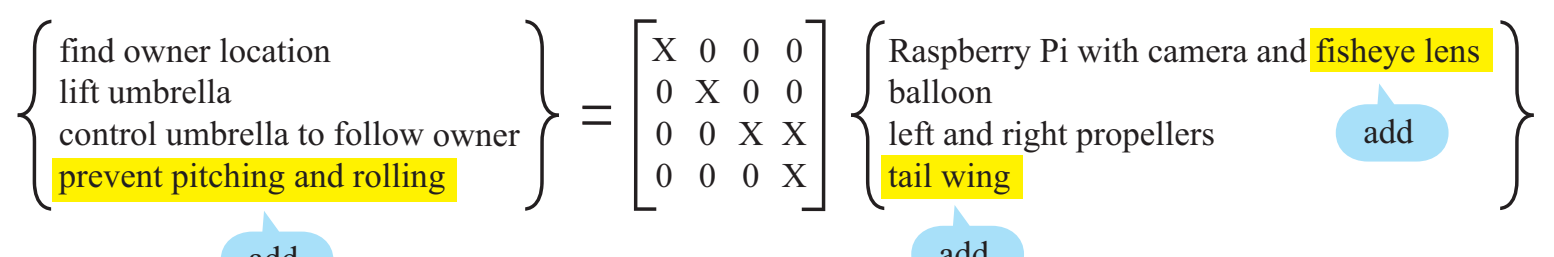
add

(d) Design equation after functional integration for FRs and physical integration for DPs

Fig. 4. Design of umbrella that follows the owner. 


\subsection{Coordinate transformation of FRs}

Students often ask us "which is a right set of FRs?" The right set of FRs is not only one. In most cases, any sets of uncoupled or decoupled designs are right because a right set of FRs can change to another right set of FRs with a thinking process of a kind of "coordinate transformation." Figure 5 shows design equations of Stirling engine. We have a design project for juniors to design and prototype a free-style Stirling engine [1], and also have them define their own design. Figure 5(b) lists FRs chronologically such as FRs of Newcomen's steam engine in Suh's textbook [2]. Figure 5(c) lists FRs functionally because the juniors have already learned the common law of heat engines. The both are correct and can improve the yellow-marked interference that a burner also heats an air cooler and the engine finally stops. This coordinate transformation will change a set of FRs and DPs, but will keep a type of design matrix like a triangular matrix in Figure 5(b) (c).

\subsection{MECE is similar to independence axiom}

The students in the School of Engineering also take the course of Methods in Business Administration for those who want to look for consultant type jobs when they graduate. The instructor of this course emphasizes that consultant has to identify a customer's problems in a mutually exclusive and collectively exhaustive (MECE) way and propose actions against problems for the customer in a one-to-one manner. This class has a higher impact and when we teach the students in the Axiomatic Design class to map one DP that satisfies a specific FR and set all FRs independently, they say they understand the concept because it is the same with MECE. Our strategy was to have the students list out all the FRs and DPs to meet the CE part of MECE using the FR-DP chart and then to make the first ME part with the design equation with $\mathrm{AD}$. When the students actually took on their projects, we found that they needed additional thinking operations of functional integration of FRs and physical integration of DPs.

\subsection{Physical integration in Suh's textbook}

Studies in Axiomatic Design has already shown the

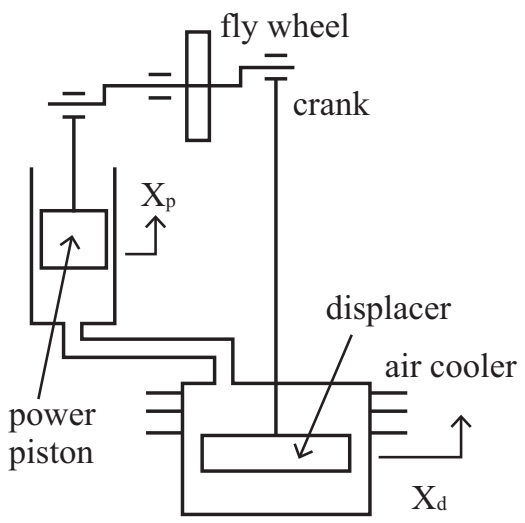

burner 0000

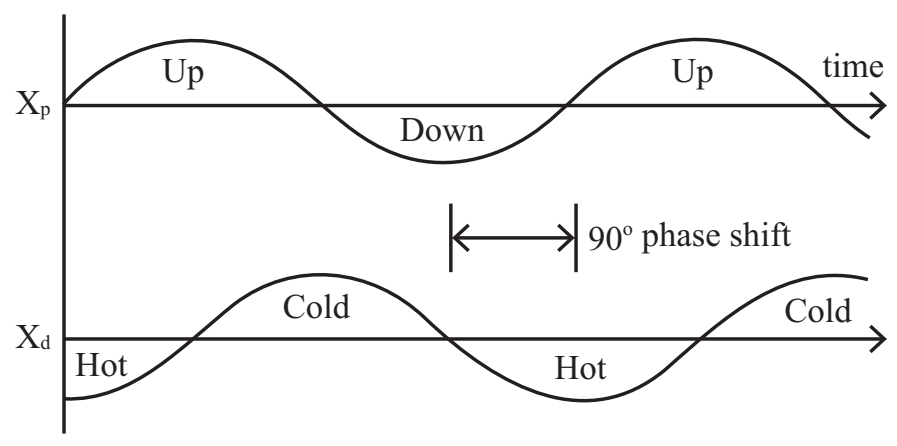

(a) Illustration of Stirling engine

$$
\left\{\begin{array}{l}
\text { generate hot air } \\
\text { push up power piston } \\
\text { generate cold air } \\
\text { pull down power piston }
\end{array}\right\}=\left[\begin{array}{llll}
\mathrm{X} & 0 & 0 & 0 \\
\mathrm{X} & \mathrm{X} & 0 & 0 \\
\mathrm{X} & 0 & \mathrm{X} & 0 \\
0 & 0 & \mathrm{X} & \mathrm{X}
\end{array}\right]\left\{\begin{array}{l}
\text { lower position of displacer at burner } \\
\text { power piston with } 90^{\circ} \text { phase shift } \\
\text { upper position of displacer at air cooler } \\
\text { inertia of fly wheel + replacement to cold air }
\end{array}\right\}
$$

(b) Design equation that list FRs chronologically

$$
\left\{\begin{array}{l}
\text { input heat } \\
\text { output heat } \\
\text { do work } \\
\text { operate cyclically }
\end{array}\right\}=\left[\begin{array}{llll}
\mathrm{X} & 0 & 0 & 0 \\
\mathrm{X} & \mathrm{X} & 0 & 0 \\
0 & 0 & \mathrm{X} & 0 \\
0 & 0 & \mathrm{X} & \mathrm{X}
\end{array}\right]\left\{\begin{array}{l}
\text { burner } \\
\text { air cooler } \\
\text { power piston with } 90^{\circ} \text { phase shift } \\
\text { displacer }+ \text { crank }+ \text { fly wheel }
\end{array}\right\}
$$

(c) Design equation that list FRs functionally

Fig. 5. FRs of Stirling engine that are listed chronologically or functionally. 
concept of physical integration. In our design engineering course, we first have the students list up FRs of PET bottles and beverage cans, as introduced in Suh's textbook [2]. The students are first surprized that the two have different physical structures, however, the 12 plus FRs are almost identical. Only the PET bottle has two additional FRs of "keep leftover drink for later" and "show contents." Also, both the beverage can and PET bottle have 12 plus FRs but they only have three physical parts of container, tab (cap), and sticker. The students then understand that each part is a result of a number of DP integrations.

\subsection{Common DP or general FR should be changed to a constraint}

When we have students construct design matrices in their design classes, the designs tend to be decoupled, and not uncoupled, because the DPs of key components or leaders related to multiple FRs. In many cases, such a long line of interferences in the design matrix cannot be improved by Axiomatic Design.

A common DP that relates to multiple FRs in a similar manner is for example, "electrical power," "control computer," "activity funds," or "related regulation." They are not key DPs for each design, however, once they are set to a DP, the corresponding column in the design matrix is filled with Xs. Similarly, a general FR like "keep low production cost," "last long," or "keep total weight light" relates to multiple DPs and the corresponding row in the design matrix will be filled with Xs. When the design has a number of such columns or rows, it is coupled and there is no room for the independence axiom. When the students come across cases with rows or columns of $\mathrm{Xs}$, they tend to underestimate the power of Axiomatic Design.

When we face such cases, we can change the constraint conditions. By setting the limits with inequalities, the relations turn into constraints and they are removed from the FR-DP relations and we can decouple the design matrix. For example, "related regulations" and "legal liabilities" for automatic driving in Fig. 1, "cost" and "quietness" for the fan in Fig. 2 or the umbrella in Fig. 4, "activity funds" for entrance exam administration in Fig. 3 can be handled as constraints. We had the errors of mixing FRs with input constraints in the Thompson's classification. [3]

\section{Conclusions}

In defining a design, we have to exhaustively list up all the FRs and DPs. Students, who are novices in design, think about FRs chronologically and about DPs spatially. If we follow the listings with design thinking of functional integration of FRs and physical integration of DPs, we can match the FR and DP counts to regularize the design matrix and it is then easier to apply Axiomatic Design. This paper introduced the listing and integration processes with simple design cases. For free subject design assignments during the courses that we taught, we found the integration to be challenging tasks for the students. Our future task is to enhance our teaching methods so all students can start design thinking of integration.

\section{References}

1. K. Iino, and M. Nakao, Design Record Graph and Axiomatic Design for Creative Design Education, PROCIR-D-16-00377, ICAD 2016 (2016)

2. N.P. Suh, Axiomatic Design, Advances and Applications (Oxford Press, 2001)

3. M.K. Thompson, Improving the requirements process in Axiomatic Design Theory, Annals of the CIRP 62(1): 115-118 (2013)

4. M.K. Thompson, A classification of Procedural Errors in the Definition of Functional Requirements in Axiomatic Design Theory, Proc. of the $7^{\text {th }}$ ICAD (2013)

5. C.A. Brown, Axiomatic Design of Manufacturing Processes Considering Coupling, Proc. of the $8^{\text {th }}$ ICAD: 149-153 (2014) 